\title{
Assembling Virtual Fixtures for Guidance in Training Environments
}

\author{
Alex B. Kuang, Shahram Payandeh, Bin Zheng, \\ Frank Henigman and Christine L. MacKenzie \\ Human Motor Systems Laboratory, School of Kinesiology \\ Experimental Robotics Laboratory, School of Engineering Science \\ Simon Fraser University \\ Burnaby, British Columbia \\ Canada, V5A 1 S6 \\ \{bkuang@,shahram@cs.,bzhenga@,frank@move.kines.,mackenzb@,sfu.ca
}

\begin{abstract}
We set up a library of virtual fixtures with both haptic and graphic properties and behaviors. For a given task, Virtual Fixture Assembly Language (VFAL) could be used to construct various virtual fixture series, with graphic and force guidance rules, making the low-level haptic and graphic rendering details transparent to the developers. An experiment evaluated the application of virtual fixtures as an aid for guiding a user in a path navigation task. The task was performed with or without force field guidance of virtual fixtures, and then transferred to the condition with no virtual fixtures. Results showed significant learning and transfer effects measured by performance time and path length. However, training using virtual fixtures with force guidance had comparable results to training with graphic only fixtures representing the path. Results are discussed in terms of motor learning theory, future work and applications for the design of better VR training environments.
\end{abstract}

\section{Introduction}

In this context, a virtual fixture is a basic unit of a computer-generated virtual tunnel with haptics and/or graphics features that is designed to provide guidance along a preferred path. These fixtures are created to enhance human performance in teleoperated tasks and virtual environments. Virtual fixtures have shown improved performance in path following experiments, through increased precision, increased speed, or both [1,2,3,4,5]. Rosenberg [2] applied haptic and auditory virtual fixtures for a three-dimensional peg-in-hole task in a teleoperation environment. He developed a general concept known as Virtual Fixturing in which force feedback information was overlaid to assist user performance in a variety of manual activities. The fixtures tested were composed of simple combinations of planar surfaces overlaid on top of the reflection of the remote task board. Experimental results showed up to a $70 \%$ improvement in performance when a virtual fixture was used compared to when no fixture was present. Park and colleagues [6] applied a virtual wall based on the location of internal mammary artery from a preoperative computed tomography scan to guide the surgeon's instrument during teleoperated coronary bypass. The virtual wall reduced execution time by over $27 \%$ and eliminated excursions beyond the desired region.

Related work on virtual fixtures also includes Cobots [7], which are robotic systems that create virtual fixtures as paths in the task space for collaborative manipulation. Cobots are most commonly used in large-scale tasks such as automobile assembly. These devices assist in power tasks and improve precision.

In summery, virtual fixtures are able to alleviate mental processing of users when they perform tasks in a $3 \mathrm{D}$ environment presented on a $2 \mathrm{D}$ screen; therefore virtual fixtures reduce the user's mental workload, and facilitate movements with higher precision requirements. In this study, we are particularly interested in investigating the role of virtual fixtures in a training environment, where a combination of virtual fixtures can guide a user through a complex scene and toward the destination, along a preferred path.

To build virtual fixtures, we used primitive shapes (i.e., sphere, cone, and cylinder) and defined geometrical and force fields within these shapes to build a virtual fixture library. When the user interacts with these shapes, he/she is able to perceive a designer specified force field through a haptic device, e.g., 3-DOF PHANToM [8]. These virtual fixtures have different attributes, behaviors and properties such as force fields which is defined within 
their constrained volumes and graphical visibilities [5]. Through assembling of different virtual fixtures and by selecting their corresponding properties, we can define a set of Virtual Fixture Assembly Language, which can be viewed as a computer-generated set of constraint rules. The experimenter has choices for assembling these fixtures for a particular task in various combinations in a serial order until a suitable combination is determined. Then, we use the final suitable assembled fixtures and apply it to define the rules for assembly of fixtures for this particular task, such that it can provide suitable guidance for the user's performance. Such constructed guidance would serve as an aid for learning a specified path.

To achieve our study goals, we built a threedimensional environment with resembles a virtual maze. In this setup, the user can feel the interaction forces of the moving object with the maze. The task for the user was to pick up a virtual object, maneuver it through the maze and place the object at a target location. We first arranged virtual fixture shapes and set up their force fields and graphics visibilities. To test for training effects, we had two practice conditions; users practised on a set of virtual fixtures with graphics visibility, and with or without force field information. Users were then transferred to a setup with no virtual fixtures -- neither graphics visibility nor force fields. The results were compared between the two virtual fixture practice conditions and across the different phases of learning and transfer.

We expected that adding virtual fixtures would be an aid to learning the task [9]. Further, virtual fixtures with both force fields and graphics visibility would facilitate learning the navigation task in a $3 \mathrm{D}$ virtual environment more than virtual fixtures with only graphics visibility. Finally, differential transfer to an environment with no virtual fixtures was predicted, such that those trained with force fields and graphics visibility would show better transfer than those trained with graphics visibility only.

\section{Virtual Fixtures}

We defined a set of Virtual Fixture Assembly Language (VFAL) and built a virtual fixture library, which allowed the virtual fixtures to have different properties and behaviors. With this language, the designer of training environments has a choice of assembling the fixtures for a particular task such that they can offer suitable guidance. With this library, force rendering can be reused without low-level programming.

\subsection{Virtual Fixture Library}

In this work, the Virtual Fixture Library is a set of the primitive shapes that have constrained attractive or repulsive force fields defined within their volumes and/or surfaces and the following behaviors.

Cone: For attractive force fields (Figure 1A), cones are always placed at the beginning of the path. They can have a larger base radius which can make them easier to locate. For example, in the current version, once the Haptic Contact Point (HCP), the tip of the PHANToM stylus, in our case, penetrates the cone surface, force cues are generated to move the HCP toward the tip. For repulsive force fields (Figure 1B), force cues are generated in such a way that once the HCP penetrates the force cone, it will be pushed away from the tip to the cone bottom.

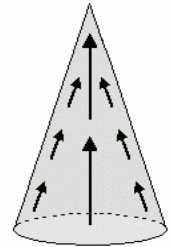

$\mathbf{A}$

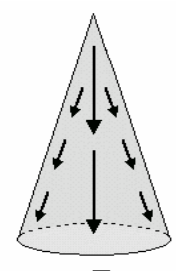

B
Figure 1. Force cone $\begin{array}{ll}\text { (A) attractive force } & \text { (B) repulsive force }\end{array}$

Sphere: For attractive force fields (Figure 2A), if the HCP penetrates the sphere surface, force cues are generated to pull the HCP to the center. This is useful to guide the user to pickup an object or place the object in a specific location. For repulsive force fields (Figure 2B), the force cue pushes away any HCP that falls inside the force sphere from the center to its surface. A sphere could be split into two parts. Each hemisphere can also have the above properties.

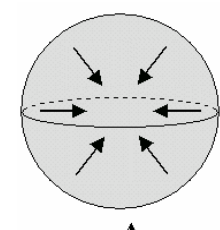

A

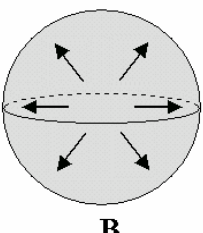

B
Figure 2. Force sphere $\begin{array}{ll}\text { (A) attractive force } & \text { (B) repulsive force }\end{array}$

Cylinder: For attractive force fields (Figure 3A), when the HCP penetrates the cylinder surface, force cues are generated to pull the HCP to the axis of the cylinder. These forces are generated perpendicular to the axis; so, the HCP is constraint to move along the axis. Furthermore, as long as the HCP enter a small area surrounding the axis, additional force cues will be generated to move the HCP toward the top of the cylinder. For repulsive force fields (Figure 3B), the cylinder works as a virtual protection tube, preventing the 
manipulated object from colliding with the protected object, which is inside the object.

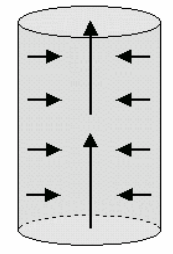

A

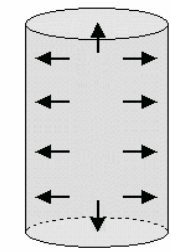

B
Figure 3. Force cylinder $\begin{array}{ll}\text { (A) attractive force } & \text { (B) repulsive force }\end{array}$

\subsection{Virtual Fixture Assembly Language}

Since we define our computer-generated constraint rules as a set of language for assembly of fixtures, there are associated "grammar" rules. To illustrate, assembling cone, cylinder, and sphere and setting up their force fields in that order will easily guide a user moving through the cone's tip and cylinder's axis and finally reach at the sphere's center. The experiment designer has the freedom to combine different force shapes to define a "virtual fixture sentence" to train the user for a particular task. However, to make the "sentence" work, he/she must obey the "grammar" rules.

In our design, the experiment designer worked with SGI Open Inventor ASCII 2.1 files (scene graph) and optional VRML files to define the VFAL. An example of defining the graphic and force properties of a virtual force cone is as follows. Lines beginning with '\#' are comments.

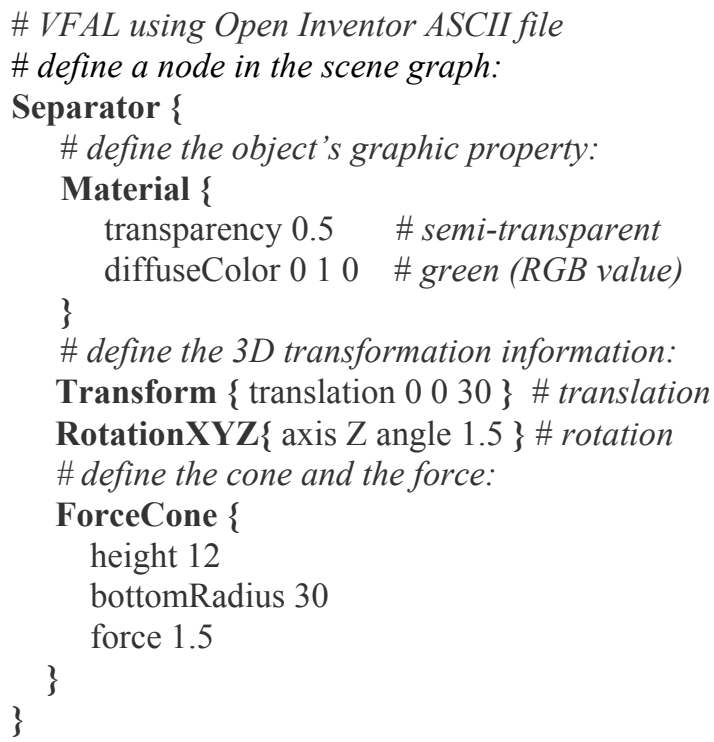

Here we also define the material properties of the force shapes using the ASCII file. Open Inventor's graphics engine provides 3D graphic rendering at $60 \mathrm{~Hz}$.
Meanwhile, PHANToM's Ghost library provides haptic rendering at $1000 \mathrm{~Hz}$.

\section{Experiment Method}

The objective of the experiment is to examine the efficiency of a telemanipulation training task with virtual fixtures. We use manipulation time and trajectory length as dependent measures.

For training, the virtual fixtures used in our experiment featured both force fields and graphics visibility or graphics visibility only. Working with the PHANToM haptic stylus, the users were required to start from a starting place, move to grab a virtual object, and maneuver it through the maze to a target location.

\subsection{Participants}

Twenty students from Simon Fraser University who volunteered to participate in the experiment were randomly assigned to two groups, the graphics and haptics $(\mathrm{GH})$ group or the graphics only $(\mathrm{G})$ group. Each group had 5 males and 5 females, ages 19 to 36 years (27 \pm 4 years) for GH group and ages 19 to 36 years $(27 \pm 4$ years) for the $G$ only group. All subjects were righthanded, had normal or corrected-to-normal vision, and had no prior knowledge of the experiment. Each subject gave informed consent and was provided with a small honorarium for 30 to 45 minutes of their time.

\subsection{Apparatus and Setup}

The experiment room was partially lighted with fluorescent lighting. On a testing table, a 17 -inch, flat monitor (Sony, Tokyo, Japan) and a PHANToM device [8] were placed as illustrated in Figure 4. The experiment software was running on Redhat Linux 7.3 on an IBM computer [10].

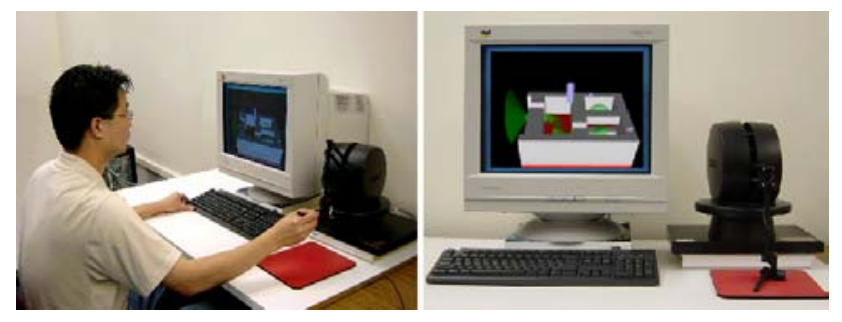

Figure 4. The experiment layout

3.2.1 Scene Description. On the monitor, a 3dimensional virtual maze was exhibited. Enclosed by a transparent glass ceiling and a red floor, the maze was separated by walls into 3 rooms (Figure 5). Throughout the entire experiment, subjects could feel the reaction 
force from interacting with the walls, ceiling and floor. Hence, haptic feedback was present always as a threedimensional force vector, as a result of the representation of the PHANToM tip and the representation of the maze.

The scene we built featured the complexity that the manipulated object must go through several narrow spaces between blocks - the gates in the walls. In this scene, the tip of the PHANToM stylus was presented by a cone colored in blue. The tip of the cone corresponded to the tip of the stylus, and the central axis of the cone was aligned with the longitudinal axis of the stylus. There was a small graphic sphere on top of the right side wall. This sphere served as the starting position for the task (see Figure 5). At the beginning of each trial, the tip of the cone was placed inside this sphere. The sphere turned light green when the stylus (represented as the cone) touched it.

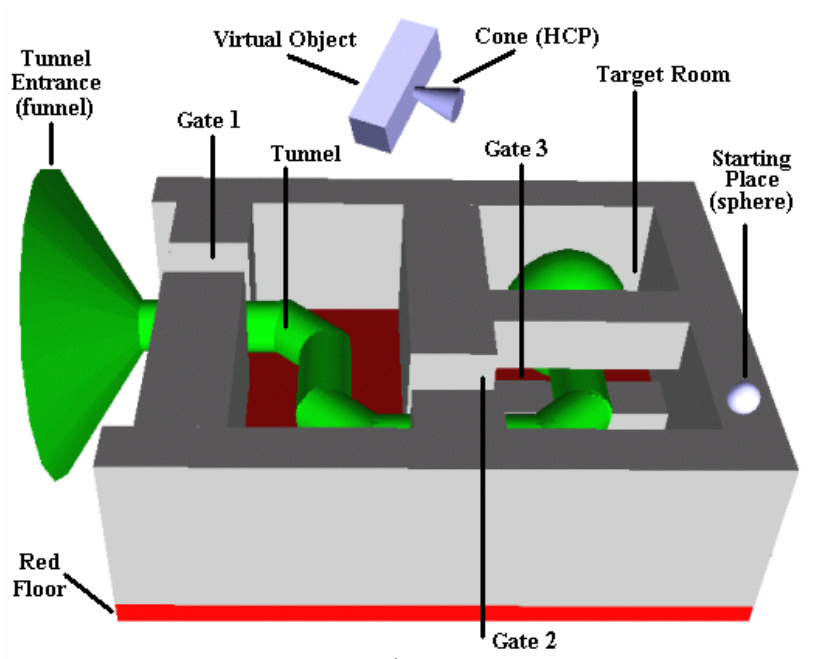

A

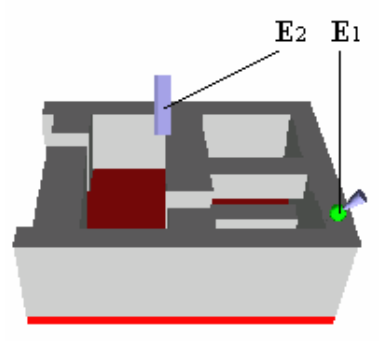

B

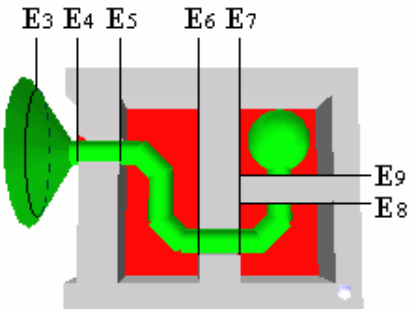

C
Figure 5

(A) Maze with green tunnel, as presented in training trials (from subject's viewing perspective)

(B) Maze with no graphic tunnel, as presented in transfer trials

(C) Events $(\mathrm{E} 1=$ Event 1, E2 = Event 2, .., E9 = Event 9)
3.2.2. The Task. Above the transparent roof was a virtual object, a 3- dimensional block (size: $12 \times 12 \times 36 \mathrm{~mm}^{3}$ ). Subjects were required to leave the starting sphere (Event 1), move the stylus cone to the virtual object and touch it. Having done so, the virtual object would be attached to the stylus cone automatically (Event 2). After that, subjects were asked to bring the virtual object to the green funnel (Event 3), pass it through gates on the walls (Event $4-8$ ) and reach the target room (Event 9). To complete the task successfully, participants had to rotate the object to match the orientation of the gates. The first and the second gates were narrow but tall, like vertical doorways (size: $30 \times 20 \times 41 \mathrm{~mm}^{3}$ ); in contrast, the third gate was wide but short (size: $70 \times 20 \times 20 \mathrm{~mm}^{3}$ ) (see Figure $5)$.

3.2.3. Experiment Design. In order to investigate how virtual fixtures help learning, subjects practiced a number of trials in this maze with a virtual tunnel added. The green tunnel connected the entrance, through the gates, to the target room in the maze. As shown in Figure 5A, the virtual tunnel was built by assembling 9 virtual fixtures. The first fixture was designed in the shape of a cone/funnel. Serving as the entrance of the tunnel, the funnel piece provided attractive forces that would drag the stylus in. Followed by the funnel were 7 cylinders that were assembled as a virtual path through the maze. This combination was chosen through initial experimentation with the assembly of fixtures. At the end of the cylinders was a sphere representing the target location. The virtual tunnel was designed to provide attractive forces to the center of the tunnel, which could be perceived by the subject by means of holding the stylus of the PHANToM. If a subject successfully moved the virtual object along the tunnel, the instantaneous force feedback generated by the virtual fixture of the tunnel kept the object inside the tunnel. As a result, fewer collisions would occur between the object and the walls, compared to the condition when no force fields were presented in the graphic only tunnel.

In our experiment, we were interested in examining the efficiency of VFAL; however, object-object collision detection and force feedback were the major problems for our implementation. This is because when two objects collide, many points are collided between these two objects and we need to design an efficient algorithm to calculate the force feedback $[11,12,13,14]$. We used an approximation algorithm - voxel sampling [15] - that applies a finite set of points on the manipulated virtual object's surface and calculated the force feedback based on collision detection between this set of points and the walls of the maze. This allowed fast approximation of collision detection and still achieved the haptic rendering rate of $1000 \mathrm{~Hz}$. 


\subsection{Procedure}

Subjects were seated comfortably on a height adjustable chair in front of the testing table, with their eyes at approximately the same height as the upper edges of the monitor. Subjects aligned the body midline with the centre of the monitor with hands resting on the table. The same instructions were read to all subjects prior to starting the experiment. They were also given a short demo to become acquainted with the use of the PHANToM stylus and the 3D virtual environment generated by the computer.

3.3.1. The Demo. The demo was provided by SensAble Inc (Boston, MA, USA). This environment contained two cubes which were placed inside a virtual room. Subjects were required to move the stylus to touch the walls and to move the cubes around inside the room. When the walls and the cubes were touched by the PHANToM stylus, the 3-dimensional force feedback provided haptic information to the subjects. Each subject interacted with this demo for less than one minute. Subjects then proceeded with our experimental task.

3.3.2. Practice and Transfer Trials. In the practice trials, subjects were exposed to the scene described in Figure 5A. Subjects initially placed the stylus to a starting sphere. When a vocal "GO" signal was given by the experimenter, subjects were required to lift the stylus from the starting sphere and move the stylus to reach the virtual object. Once the stylus touched the object, the object automatically attached to the stylus cone. Then, the subjects transported the virtual object to the entrance the funnel piece of the green tunnel. Hereafter, subjects were required to move the virtual object as carefully and quickly as possible through the gates in the walls to the target in the back room. Subjects were instructed that they should use the green tunnel as guidance; however, the complete submerging of the virtual object inside the tunnel was not necessary. A trial was terminated and treated as successful trial when the entire body of virtual object was in the target room.

Twenty subjects were randomly assigned to either the $\mathrm{GH}$ group or $\mathrm{G}$ group for the practice section. In the $\mathrm{GH}$ group, the tunnel exhibited both green graphic (diffuse color $=\operatorname{RGB}(0,0.6,0)$, transparency $=0.5)$ and haptic (attractive force $=0.6$ Newton) guidance features. Subjects were able to see this green tunnel and perceive the force fields when carrying the object through the tunnel. In contrast, in the G group, subjects could only see the green tunnel (diffuse color $=\operatorname{RGB}(0,0.6,0$ ), transparency $=0.5$ ) but were not able to feel any force fields (force $=0$ Newton) of the tunnel. Note however that all subjects received force feedback from contacting walls, ceiling or floor. For each subject, a total of 15 practice trials were performed before 5 transfer trials.

For transfer trials the maze was displayed without the green tunnel or force fields (see Figure 5B). Subjects were informed that they were continuing to do the same task under a slightly different virtual environment. The task and the requirements were maintained the same as they were in the practice section. Each subject performed 5 trials in the transfer portion of the experiment.

3.3.4. Data Analysis. When the stylus cone broke contact with the starting sphere, this event was recorded by the computer. The second event was the moment when the stylus touched the virtual object. For data analysis, seven virtual planes were implanted at the following locations: a) entrance of the funnel, b) entrance to the $1^{\text {st }}$ gate, c) exit from the $1^{\text {st }}$ gate, d) entrance to the 2 nd gate, e) exit from the $2^{\text {nd }}$ gate, f) entrance to the $3^{\text {rd }}$ gate, g) exit from the $3^{\text {rd }}$ gate. When the tip of PHANToM stylus first contacted these virtual planes, the computer stored times and the 3D positions of the stylus tip. By subtracting the time between the abovementioned events, movement durations were obtained for each phase, for each trial: Time 1, acquire object; time 2, transport object to funnel; time 3 , to enter gate 1 ; time 4 , to exit gate 1 ; time 5 , to enter gate 2 ; time 6 , to exit gate 2 ; time 7 , to enter gate 3 ; time 8 , to exit gate 3 ; and total task time.

By calculating the path distance that the PHANToM stylus travelled between each of those events, movement path lengths were obtained from each trial: path 1, acquire object; path 2, transport object to funnel; path 3, enter gate 1 ; path 4 , exit gate 1 ; path 5 , enter gate 2 ; path 6 , exit gate 2 ; path 7 , enter gate 3 ; path 8 , exit gate 3 ; and total task path.

In order to dissect the learning process, we blocked the 15 practice trials into 3 phases, i.e., the initial phase of trials $1-5$, the middle phase of trials $6-10$, and the late phase of trials $11-15$. We included a transfer phase for trials $16-20$. Temporal and trajectory measures were computed. Data were analyzed with SPSS 11.0 (SPSS Inc, Chicago, Illinois, USA) by a 2 (groups) x 4 (learning phases) mixed factorial design model, with repeated measures on the second factor.

Means and standard errors are reported for significant effects, with an a priori alpha level of 0.05

\section{Experiment Results}

We measured performance in terms of manipulation time and trajectory path length. The results and analysis are given as follows. 


\subsection{Temporal measures}

No practice group effect was revealed. However, learning effects were found for time $1\left(\mathrm{~F}_{(3,54)}=17.957, \mathrm{p}\right.$ $<0.001)$, time $2\left(\mathrm{~F}_{(3,54)}=17.686, \mathrm{p}<0.001\right)$ time $4\left(\mathrm{~F}_{(3,}\right.$, ${ }_{54)}=5.604, \mathrm{p}=0.011$, time $5\left(\mathrm{~F}_{(3,54)}=16.970, \mathrm{p}<0.001\right)$, time $7\left(\mathrm{~F}_{(3,54)}=12.676, \mathrm{p}<0.001\right.$, and total time $\left(\mathrm{F}_{(3,54)}\right.$ $=10.590, \mathrm{p}=0.002$ ).

As shown in table 1, subjects took longest time in the initial phase of practice. Movement times significantly dropped as the practice continued. In transfer trials, movement time increased to some extent; however, total time of transfer trials was still better than in the initial training phase (Figure 7).

\subsection{Trajectory measures}

By analyzing the trajectory path length measures, no training group effect was revealed. However, learning effects were found for path $2\left(\mathrm{~F}_{(3,54)}=9.736, \mathrm{p}<0.001\right)$, path $5\left(\mathrm{~F}_{(3,54)}=7.042, \mathrm{p}=0.003\right)$, path $7\left(\mathrm{~F}_{(3,54)}=7.898\right.$, $\mathrm{p}=0.004$, and total path $\left(\mathrm{F}_{(3,54)}=4.907, \mathrm{p}=0.021\right)$.

Table 1. Mean and SE of times (ms) in both the practice and transfer trials. Significant learning effects are indicated by *

\begin{tabular}{lrrrr}
\hline & & Practice & & Transfer \\
& $\begin{array}{r}\text { initial } \\
\text { phase }\end{array}$ & $\begin{array}{r}\text { middle } \\
\text { phase }\end{array}$ & $\begin{array}{r}\text { late } \\
\text { phase }\end{array}$ & $\begin{array}{r}\text { transfer } \\
\text { phase }\end{array}$ \\
\hline time 1* & 1677 & 1182 & 1096 & 1130 \\
& 147 & 113 & 103 & 79 \\
time 2* & 3318 & 2560 & 2465 & 2377 \\
& 194 & 132 & 155 & 209 \\
time 3 & 5491 & 3245 & 2445 & 4324 \\
& 1716 & 1150 & 796 & 1258 \\
time 4* & 1314 & 594 & 555 & 694 \\
& 270 & 127 & 79 & 130 \\
time 5* & 3273 & 1976 & 1868 & 1921 \\
& 411 & 252 & 173 & 218 \\
time 6 & 3335 & 1337 & 827 & 715 \\
& 1401 & 409 & 193 & 131 \\
time 7* & 2528 & 1466 & 1324 & 1566 \\
& 343 & 173 & 166 & 208 \\
time 8 & 986 & 783 & 648 & 632 \\
& 177 & 187 & 104 & 137 \\
total time* & 21922 & 13143 & 11230 & 13359 \\
& 2975 & 1851 & 1267 & 1850 \\
\hline
\end{tabular}

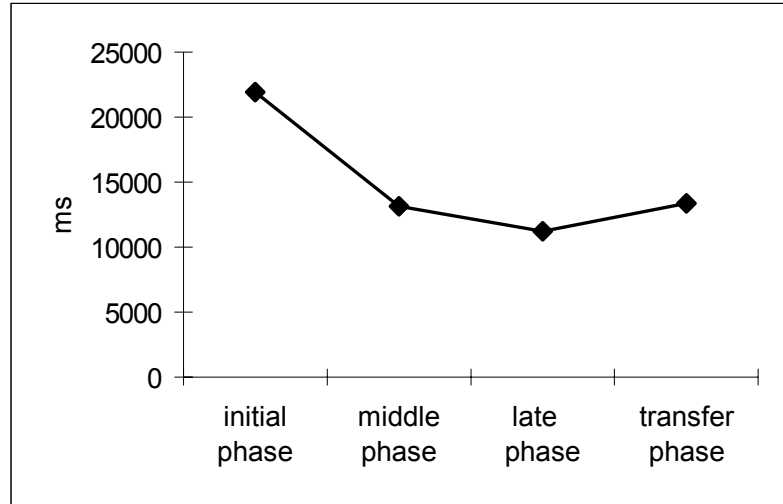

Figure 7. Total task time (ms) across learning and transfer phases.

As indicated in table 2, subjects took the longest paths in the initial phase of the practice trials. Movement path significantly dropped as the practice continued. When performing in the transfer, movement paths bounced up slightly (except for path 2); however, the total path took in the transfer was shorter than in the initial practice phase (Figure 8).

\section{Table 2. Mean and SE of paths $(\mathrm{mm})$ in both the practice and transfer trials. Significant learning effects are indicated by *}

\begin{tabular}{lrrrr}
\hline & $\begin{array}{r}\text { Practice } \\
\text { initial } \\
\text { phase }\end{array}$ & $\begin{array}{r}\text { middle } \\
\text { phase }\end{array}$ & $\begin{array}{r}\text { late } \\
\text { phase }\end{array}$ & $\begin{array}{r}\text { Transfer } \\
\text { transfer } \\
\text { phase }\end{array}$ \\
\hline path 1 & 200 & 184 & 179 & 186 \\
& 10 & 7 & 6 & 6 \\
path 2* & 292 & 293 & 292 & 254 \\
& 8 & 9 & 10 & 8 \\
path 3 & 296 & 191 & 143 & 246 \\
& 94 & 66 & 38 & 58 \\
path 4* & 81 & 44 & 45 & 53 \\
& 16 & 6 & 5 & 7 \\
path 5* & 165 & 136 & 134 & 142 \\
& 12 & 7 & 5 & 6 \\
path 6 & 161 & 86 & 64 & 56 \\
& 55 & 21 & 17 & 10 \\
path 7* & 150 & 102 & 89 & 114 \\
& 20 & 7 & 6 & 11 \\
path 8 & 61 & 52 & 52 & 48 \\
& 12 & 9 & 7 & 10 \\
total path* & 1406 & 1089 & 998 & 1100 \\
& 136 & 86 & 49 & 74 \\
\hline & & & &
\end{tabular}




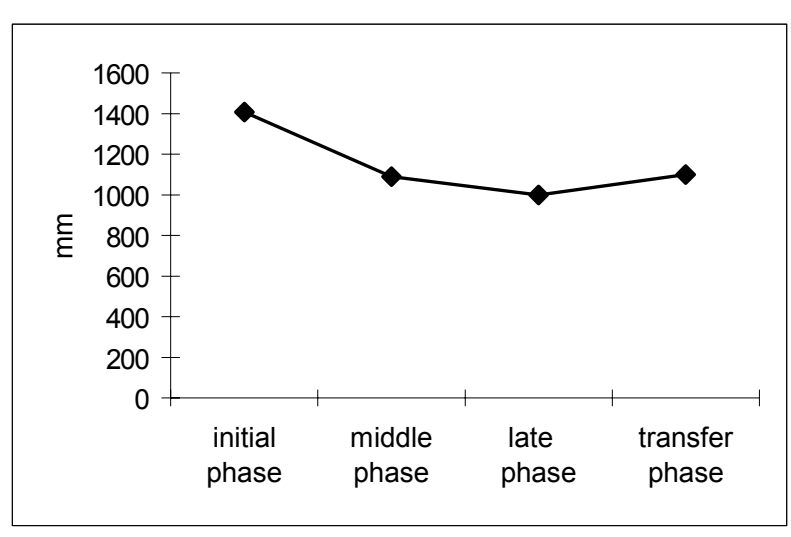

Figure 8. Total path length $(\mathrm{mm})$ across learning and transfer phases.

\section{Discussion}

In order to explore the usefulness and usability of virtual fixtures as a guide in training environments, we developed a Virtual Fixture Assembly Language (VFAL), and a Virtual Fixture Library. Virtual fixtures were developed from primitive geometric shapes, with a variety of graphic and haptic properties and behaviors. Our view is that the designer of training environments could select the nature and assembly order of such virtual fixtures, to provide sufficient, necessary or optimal guidance in learning a teleoperated task. For example, in designing our environment, we included a cone, cylinders and a sphere, with graphics visibility, and in some cases, attractive force fields, to provide guidance along the maze path. Our initial fixtures had only 4 cylinders inside the building; pilot work in planning the experiment with this sequence indicated a high occurrence of collisions with the inner walls of the maze. So our next iteration of virtual fixtures used 7 cylinders instead of 4 cylinders between the entry cone (funnel) and ending sphere, for "rounding the corners", and reducing wall collisions. In this paper, we used only one specific sequence of assembled virtual fixtures, and varied whether or not those fixtures had graphics visibility only $(G)$ or both graphics visibility and attractive force fields $(\mathrm{GH})$. Of course, many other computer-generated display options, fixture types, and assembly sequences of virtual fixtures are possible. Also, for this initial study, we use a simple experimental environment for studying the effectiveness of Virtual Fixtures. For the future work, we can incorporate CAD models and implement general collision detection approaches.

Our experiment investigated the learning and transfer of maze navigation with a single specified assembly of virtual fixtures. As expected, we found typical learning effects in the maze traversal task [16]: as shown in Figures 7 and 8 , both total task time and path length decreased systematically from initial through middle to later phases of learning. In transferring to a no-fixture condition for the maze navigation, results showed some transfer of learning on the maze, for both task time and spatial path measures. Note that this transfer was to a different implementation under the same virtual reality environment. However, there were no differential effects between the Graphics only $(\mathrm{G})$ and Graphics plus force fields $(\mathrm{GH})$ groups. Contrary to our prediction, the learning effects did not differ significantly between the graphics only $(G)$ and graphics plus force fields groups $(\mathrm{GH})$, nor was there an advantage for the GH group on the transfer trials. Thus graphic tunnel information alone was as useful as graphic and force field guidance through the tunnel. Given that subjects had to navigate a spatial path, and orient the virtual object accordingly to maneuver through the gates, it may be that the graphic path information, combined by haptic feedback received by both groups when they collided with walls, was sufficient for performance of the task. Based on this graphic feedback, and haptic collision information, subjects had a good representation of where they were in the virtual environment and what they needed to do to progress through and complete the maze. The force fields of the virtual fixture (the tunnel), for this scene and task in this particular experiment, provided no added benefit. In the future work, we will design an experiments where the effect of haptic from walls, floor and ceiling will be taken away; providing a scenario that does not have any haptic feedback to further study the issue. Also, had we provided 6-DOF force guidance with the PHANToM stylus, subjects may have more easily rotated the virtual object to maneuver through the gates. However, the results and conclusions of this paper can apply for any types of haptic feedback devices because our objective is to explore the effectiveness of virtual fixtures. Finally, since we only tested one specific assembly of virtual fixtures, it remains an open question whether there is a most suitable fixture sequence for learning this task, and what that fixture assemblage entails.

Future work could address different geometries for virtual fixtures, different assemblages or layouts of virtual fixture sequences, and different modality combinations for computer-generated displays and guidance in training environments. Under what conditions is haptic guidance through attractive and repulsive force fields beneficial and under what conditions is such guidance detrimental? Research with human users could address the following issues: What task requirements and characteristics are various approaches to virtual fixtures suitable and beneficial? What are the necessary and sufficient conditions for virtual fixtures to be useful? For training environments, are there differential retention and transfer effects of learning? Do advantages gained in virtual 
training environments generalize to performance of real world tasks? How veridical must display modalities be for such transfer? Is training for a single individual different from team training for Computer-supported Collaborative Work? Is there one optimum approach to virtual fixtures for training, or could there be allowances for individual differences in learning styles, to be specified by the users based on their preferences?

\section{References}

[1] P. Marayong, A. Bettini, and A.M. Okamura, "Effect of Virtual Fixture Compliance on Human-Machine Cooperative Manipulation", IEEE/RSJ Int'l. Conf. on Intelligent Robots and Systems, 2002, pp. 1089-1095.

[2] L. Rosenberg, "Virtual Fixtures: Perceptual Tools for Telerobotic Manipulation”, Proceedings of IEEE Virtual Reality Int'l. Symposium, 1993, pp. 76-82.

[3] A. Bettini, S. Lang, A. Okamura, and G. Hager, "Vision Assisted Control for Manipulation Using Virtual Fixtures: Experiments at Macro and Micro Scales", Proceedings of the IEEE International Conference on Robotics and Automation, 2002, pp. 3354-3361.

[4] A. Bettini, S. Lang, A. Okamura and G. Hager, "Vision Assisted Control for Manipulation Using Virtual Fixtures", IEEE/RSJ International Conference on Intelligent Robots and Systems, 2001, pp. 1171-1176.

[5] S. Payandeh and Z. Stanisic, "On Application of Virtual Fixtures as an Aid for Telemanipulation and Training", Symposium on Haptic Interfaces for Virtual Environment and Teleoperator Systems, 2002, pp. 18-23.

[6] S. Park, R. Howe, and D. Torchiana, "Virtual fixtures for Robotic Cardiac Surgery", 4th International Conference on Medical Image Computing and Computer-Assisted Intervention, Utrecht, The Netherlands, 2001, pp. 14-17.

[7] M. Peshkin, C. Moore, B. Gillespie, and J.E. Colgate, "Cobot Architecture", IEEE Transactions on Robotics and Automation, 2001, Vol. 17, No. 4, pp. 377-390.

[8] PHANToM Premium 1.5, an 3-D haptics device with 3-DOF force feedback and 6-DOF position sensing, produced by SensAble Technologies, Inc. (Boston, MA, USA).

[9] D. Feygin, M. Keehner, and F. Tendick, "Haptic Guidance: Experimental Evaluation of a Haptic Training Method for a Perceptual Motor Skill", Proceedings 10th Symposium on Haptic Interfaces for Virtual Environment and Teleoperator Systems, Orlando, FL, 2002, pp. 40--47.

[10] IBM Intellistation M Pro computer (Markham, Ontario, Canada): dual Xeon $2.4 \mathrm{GHz}$ processor, $512 \mathrm{MB}$ RAM, NVIDIA Quadro4 900XGL graphics card.
[11] A.D. Gregory, M.C. Lin, S. Gottschalk, and R. Taylor, "Fast and accurate collision detection for haptic interaction using a three degree-of-freedom force-feedback device", Computational Geometry, 2000, Vol. 15, No. 1-3, pp. 69-89.

[12] Y.J. Kim, M.C. Lin and D. Manocha, "DEEP: Dual-space Expansion for Estimating Penetration Depth between convex polytopes", Robotics and Automation, IEEE International Conference, 2002, Vol. 1, pp. 921 -926.

[13] M.K. Ponamgi, D. Manocha, and M.C. Lin, "Incremental algorithms for collision detection between polygonal models", Visualization and Computer Graphics, IEEE Transactions, 1997, Vol. 3, Issue 1, pp. $51-64$.

[14] M.C. Lin, D. Manocha, and M. Ponamgi, "Fast algorithms for penetration and contact determination between non-convex polyhedral models", Robotics and Automation, IEEE International Conference, 1995, Vol. 3, pp. 2707 -2712.

[15] W. McNeely, K. Puterbaugh, and J. Troy. "Six degree-offreedom haptic rendering using voxel sampling". Proc. of ACM SIGGRAPH, 1999, pp 401-408.

[16] R.A. Schmidt and T.D. Lee, Motor control and learning: a behavioral emphasis, Human Kinetics Publishers, Champaign, Illinois, 1999. 